\title{
More on the Analysis of Dilute Solution Data: Polystyrenes Prepared Anionically in Tetrahydrofuran
}

\author{
Akihiko Yamamoto, Motoharu Fujil, Genzo Tanaka, \\ and Hiromi YamaKawA* \\ Department of Polymer Chemistry, Kyoto University, \\ Kyoto, Japan.
}

(Received June 19, 1971)

\begin{abstract}
Further comments on the analysis of dilute solution data are provided. Light-scattering and intrinsic-viscosity data used for this purpose are those obtained for monodisperse polystyrenes, prepared anionically in tetrahydrofuran, in benzene, toluene, and dichloroethane at $30^{\circ} \mathrm{C}$ and in cyclohexane at temperatures ranging from 32.2 to $60.1^{\circ} \mathrm{C}$. The value of $[\eta]_{\theta} / M^{1 / 2}$ is somewhat greater than the corresponding value for Berry's polystyrenes prepared anionically in benzene, where $[\eta]_{\theta}$ is the intrinsic viscosity at the theta temperature and $M$ is the polymer molecular weight. This suggests that the two types of samples differ in microstructure, though the precise difference is unknown. However, it is shown that the two-parameter relationships established experimentally in the previous papers are well reproduced in the present data. The relationships are different from those determined by Kato, et al., for monodisperse poly $(\alpha$-methylstyrene) prepared anionically. Since there is shown to be no essential difference between our and their methods of determining mean-square radii, it seems unlikely that the difference is related to measurements and subsequent treatments. It is suggested rather that the problem is related to Kato's samples.

KEY WORDS Two-parameter Theory / Excluded-volume Effect / Mean-square Radius / Second Virial Coefficient / Intrinsic Viscosity / Expansion Factor / Scattering Function / Polystyrene / Anionic Polymerization /
\end{abstract}

In the previous paper, ${ }^{1}$ it has been pointed out that recent experimental studies on the excluded-volume effect in dilute polymer solutions may be classified into two groups with respect to the behavior of experimental data. The investigations of Berry, ${ }^{2}$ Norisuye, et al., ${ }^{3,4}$ and Tanaka, et al., ${ }^{1,5}$ belong to the same category, which will be referred to as the first group, and the work of Kato, et al., ${ }^{6,7}$ is representative of the second group. There is fairly close agreement between the results of the two groups for theta-solvent systems, while the difference between them is noticeable for good-solvent systems, i.e., for large expansion factor $\alpha_{\mathrm{S}}$, as defined by $\alpha_{\mathrm{s}}{ }^{2}=\left\langle S^{2}\right\rangle \mid\left\langle S^{2}\right\rangle_{0}$, where $\left\langle S^{2}\right\rangle$ is the mean-square radius of the polymer chain and $\left\langle S^{2}\right\rangle_{0}$ is its unperturbed value in the theta state. The different points are summarized as follows. (1) Observed values of the interpenetration func-

\footnotetext{
* To whom correspondence should be addressed.
}

tion $\Psi$ appearing in the second virial coefficient $A_{2}$ are $0.25-0.30$ for large $\alpha_{S}$ is the first group, while the corresponding values reach only 0.20 in the second, where $\Psi$ is defined by

$$
\Psi=A_{2} M^{2} / 4 \pi^{3 / 2} N_{A}\left\langle S^{2}\right\rangle^{3 / 2}
$$

with $M$ the polymer molecular weight and $N_{A}$ the Avogadro number. (2) Observed values of the viscosity-radius expansion factor $\alpha_{\eta}$, as defined by $\alpha_{\eta}^{3}=[\eta] /[\eta]_{\theta}$ with $[\eta]$ the intrinsic viscosity and $[\eta]_{\theta}$ its value in the theta state, depend only on the excluded-volume parameter $z$ in the first group, while this is not the case in the second, where $z$ is defined by

$$
z=\left(4 \pi\left\langle S^{2}\right\rangle_{0}\right)^{-3 / 2} \beta n^{2}
$$

for the chain of $n$ effective bonds with $\beta$ the binary-cluster integral for a pair of segments. Note that Berry's viscosity data ${ }^{2}$ proved to belong rather to the first group after the reanalysis by 
Fujita, et al. ${ }^{4}$

Possible reasons for these differences are as follows. (1) The polymer samples used in the first group are not always monodisperse, while Kato's samples, poly( $\alpha$-methylstyrene), may be regarded as quite monodisperse. (2) The two groups have used different methods of determining the mean-square radius $\left\langle S^{2}\right\rangle$ from raw lightscattering data. The first group has applied the so-called square-root plot, while Kato, et al., have determined $\left\langle S^{2}\right\rangle$ to force agreement between observed and theoretical values of the scattering function (angular distribution).

The two groups have used different methods of analyzing data to examine the agreement between the two-parameter theory and experiment. The first point requires some comments. It has been believed that if the samples are polydisperse, rather low values of $\Psi$ are obtained, because the $z$-average of $\left\langle S^{2}\right\rangle$ is greater than the weight average. Indeed, this has been explicitly shown in the case of poly( $p$-methylstyrene). ${ }^{5}$ However, the effect should be reexamined in relation to the second point. It is the purpose of this paper to make further investigation of the problem, directing attention to the three points cited above.

For this purpose, we obtained light-scattering and viscosity data for monodisperse polystyrenes in theta and good solvents. However, since Berry $^{2}$ already used monodisperse polystyrenes, which were prepared by anionic polymerization in benzene, ${ }^{8}$ we prepared our samples anionically in tetrahydrofuran. In anticipation of the results obtained, it must be noted that there are recognizable differences in the values of $[\eta]_{\theta} / M^{1 / 2}$ between Berry's and our samples. Further, we chose cyclohexane as a theta solvent, and benzene, toluene, and dichloroethane as good solvents to examine the behavior of data in detail in the range of large $\alpha_{\mathrm{S}}$. As a result of the analysis of the present data, it will again be emphasized that a two-parameter theoretical description of the behavior of dilute polymer solutions is valid as a first approximation or within experimental uncertainty, and that the $z$-dependences of the related quantities are well reproduced. We shall also discuss methods of estimating the binary-cluster integral from light- scattering and viscosity data.

\section{EXPERIMENTAL}

\section{Materials}

Polystyrene was prepared by anionic polymerization at $-78^{\circ} \mathrm{C}$ (with a Dry Ice-methanol mixture) according to the procedure of Morton, et al. ${ }^{9,10}$ using tetrahydrofuran as a solvent and $n$-butyllithium as an initiator. The termination of polymerization was achieved with a methanol -butanol mixture.

Each of the whole polymers thus obtained was divided into three fractions by the addition of methanol to its $0.1-0.3-\%$ solution in toluene at $30^{\circ} \mathrm{C}$, and five of the middle fractions were used as samples for light-scattering and viscosity measurements. Each sample was dissolved in toluene, filtered through a $30-\mu$-sintered glass filter, precipitated into methanol, and dried by evacuation at $50^{\circ} \mathrm{C}$. Sedimentation patterns taken for two of the samples in cyclohexane at $35^{\circ} \mathrm{C}$ both led to values for $M_{w} / M_{n}$ of less than 1.06, where $M_{w}$ and $M_{n}$ are the weightaverage and number-average molecular weights of a given sample, respectively. The samples may therefore be expected to have very sharp distributions in molecular weight.

Light-scattering and viscosity measurements were carried out in good solvents at $30^{\circ} \mathrm{C}$, and only on one sample in cyclohexane at temperatures ranging from 32.2 to $60.1^{\circ} \mathrm{C}$ and on other samples at the $\Theta$ temperature. All the solvents were purified according to standard procedures, and fractionally distilled. For the densities of solvents at temperatures of measurement, literature values ${ }^{11}$ were used. Refractive indices of these solvents for light of wavelength $436 \mathrm{~m} \mu$ were calculated according to the same procedure as before. ${ }^{1,5}$ The results thus obtained at $30^{\circ} \mathrm{C}$ are $1.515_{5}$ for benzene, $1.513_{9}$ for toluene, and $1.449_{8}$ for dichloroethane. For cyclohexane, the values at $32.2,40.2$, and $60.1^{\circ} \mathrm{C}$ are $1.428_{3}$, $1.423_{5}$, and $1.411_{2}$, respectively.

The most concentrated solutions of the polymers in benzene, toluene, and dichloroethane were stirred continuously for one day at room temperature and sequential dilutions were made to obtain test solutions. Dichloroethane solutions were handled in the dark as much as 
possible to prevent photo and oxidative degradation of the polymer. The most concentrated solution of each sample in cyclohexane was stirred continuously for $4-7$ days at $47^{\circ} \mathrm{C}$ and sequential dilutions were made. The concentrations of the most concentrated solutions were determined gravimetrically, and those of all the diluted solutions were determined from known dilution factors.

\section{Light Scattering}

Light-scattering measurements were carried out in a Shimazu photometer with a cylindrical cell. The apparatus was calibrated according to the same procedure as before, ${ }^{1,5}$ taking the Rayleigh ratio of pure benzene at $30^{\circ} \mathrm{C}$ as $49.5 \times 10^{-6}$ for light of wavelength $436 \mathrm{~m} \mu .^{12}$ Optical purification of both the most concentrated solutions and solvents was carried out by centrifugation at $20000 \mathrm{rpm}$ for $1 \mathrm{hr}$ using a Marusan centrifuge. Each purified cyclohexane solution was stirred in a cell at $40^{\circ} \mathrm{C}$ for one day before measurements. All measurements were carried out with unpolarized light of wavelength $4.36 \mathrm{~m} \mu$, and data were obtained for four or five polymer concentrations for scattering angles from 35 to $145^{\circ}$. For solutions of the sample with the highest molecular weight $\left(M_{w} \simeq 2.9 \times 10^{6}\right)$ in good solvents, the range of concentration was made as low as possible (down to $c a .0 .05 \mathrm{~g} / \mathrm{d} l$ ). The sample temperature was held to $\pm 0.05^{\circ} \mathrm{C}$ over the range of temperature from 30 to $60.1^{\circ} \mathrm{C}$.

Refractive-index-increment measurements were carried out at $30^{\circ} \mathrm{C}$ using a Shimazu differential refractometer. The results for $436 \mathrm{~m} \mu$ light were $0.115(\mathrm{~m} / \mathrm{g})$ for benzene and 0.166 for dichloroethane. For toluene, the literature value $(0.118)^{13}$ was adopted. For cyclohexane, measurements were not carried out for the reason stated below.

Treatments of all scattering data were made according to the procedure of Berry, ${ }^{2}$ plotting the square root of $K c / R_{\theta}$ against $c$ and $\sin ^{2}(\theta / 2)$ with $R_{\theta}$ the Rayleigh ratio, $c$ the polymer concentration in conventional units, $\theta$ the scattering angle, and $K$ the well-known constant involving the refractive index and its increment. The procedure of Fujita ${ }^{14}$ was also applied to determine $\left\langle S^{2}\right\rangle$ for the sample with the highest molecular weight in good solvents. Values of
$M_{w}$ were not obtained in cyclohexane, since the calibration constant for the apparatus was available at $30^{\circ} \mathrm{C}$ but measurements on cyclohexane solutions could not be carried out at this temperature. For these solutions, second virial coefficients were determined from the ratios of slopes to intercepts of const. $\left(c / R_{\theta}\right)^{1 / 2} v s$. $c$ (which are equal to $A_{2} M_{w}$ ), using values of $M_{w}$ obtained in benzene.

\section{Viscosity}

Viscosity measurements were carried out using a viscometer of the Ubbelohde type at the same temperatures as those for the light-scattering measurements. The sample temperature was held to $\pm 0.02^{\circ} \mathrm{C}$. Kinetic-energy corrections were unnecessary. Shear-rate correction were unnecessary except for solutions of the sample with the highest molecular weight in good solvents, for which indirect corrections were applied (see the next section). The data were extrapolated to infinite dilution to determine $[\eta]$, plotting $\eta_{\mathrm{sp}} / c$ against $c$, and also $\left(\ln \eta_{\mathrm{r}}\right) / c$ against $c$ for cyclohexane solutions, where $\eta_{\mathrm{sp}}$ and $\eta_{\mathrm{r}}$ are the specific and relative viscosities of the solutions, respectively.

\section{RESULTS}

Tables I to III summarize light-scattering and intrinsic-viscosity data for five polystyrene samples in benzene, toluene, and dichloroethane, respectively, at $30^{\circ} \mathrm{C}$. Figure 1 shows $\log -\log$ plots of $\left\langle S^{2}\right\rangle$ against $M_{w}$ for polystyrene in these good solvents. The equations of straight lines in the figure are

$$
\begin{array}{ll}
\left\langle S^{2}\right\rangle=1.92 \times 10^{-18} M_{w}{ }^{1.17} & \text { (in benzene at } \left.30^{\circ} \mathrm{C}\right) \\
\left\langle S^{2}\right\rangle=2.05 \times 10^{-18} M_{w}{ }^{1.16} & \text { (in toluene at } \left.30^{\circ} \mathrm{C}\right) \\
\left\langle S^{2}\right\rangle=1.89 \times 10^{-18} M_{w}{ }^{1.16} & \text { (in dichloroethane at } \\
& \left.30^{\circ} \mathrm{C}\right)
\end{array}
$$

which were determined by the method of least squares, $\left\langle S^{2}\right\rangle$ being expressed in centimeters squared. Figure 2 shows $\log$-log plots of $[\eta]$ against $M_{w}$ for the same systems. Each straight line was determined by applying the method of least squares to the four data points, excluding the highest. The equations of lines are

$$
\begin{aligned}
& {[\eta]=1.15 \times 10^{-4} M_{w}{ }^{0.73} \quad\left(\text { in benzene at } 30^{\circ} \mathrm{C}\right)} \\
& {[\eta]=8.81 \times 10^{-5} M_{w}{ }^{0.75} \quad\left(\text { in toluene at } 30^{\circ} \mathrm{C}\right)}
\end{aligned}
$$




$$
[\eta]=8.38 \times 10^{-5} M_{w}^{0.74} \quad \begin{aligned}
& \text { (in dichloroethane at } \\
& 30^{\circ} \mathrm{C} \text { ) }
\end{aligned}
$$

where $[\eta]$ is expressed in deciliters per gram.

Berry $^{2}$ has also plotted $\log [\eta]$ against $\log M$ over a much wider range of $M$ for polystyrene in toluene, using his own data $\left(\right.$ at $12.2^{\circ} \mathrm{C}$ ) and

Table I. Light-scattering and intrinsic-viscosity data for polystyrene in benzene at $30^{\circ} \mathrm{C}$

\begin{tabular}{rcccl}
\hline Samples & $M_{w} \times 10^{-4}-\begin{array}{c}A_{2} \times 10^{4}, \\
\mathrm{~m} l \mathrm{~mol} / \mathrm{g}^{2}\end{array}$ & $\begin{array}{c}\left\langle S^{2}\right\rangle \times 10^{12} \\
\mathrm{~cm}^{2}\end{array}$ & $\begin{array}{l}{[\eta],} \\
\mathrm{d} l / \mathrm{g}\end{array}$ \\
\hline VIII & 287 & 2.25 & 68.7 & $5.83(6.17)^{\mathrm{a}}$ \\
II & 159 & 2.91 & 33.1 & 3.99 \\
VII & 75.6 & 3.34 & 14.0 & 2.30 \\
V & 34.2 & 4.12 & 5.74 & 1.31 \\
III & 24.6 & 4.46 & 3.80 & 1.01 \\
\hline
\end{tabular}

${ }^{a}$ Extrapolated to zero rate of shear (see the text).

Table II. Light-scattering and intrinsic-viscosity data for polystyrene in toluene at $30^{\circ} \mathrm{C}$

\begin{tabular}{rcccl}
\hline Samples & $M_{w} \times 10^{-4}$ & $\begin{array}{c}A_{2} \times 10^{4}, \\
\mathrm{~m} l \mathrm{~mol} / \mathrm{g}^{2}\end{array}$ & $\begin{array}{c}\left\langle S^{2}\right\rangle \times 10^{12} \\
\mathrm{~cm}^{2}\end{array}$ & $\begin{array}{l}{[\eta],} \\
\mathrm{d} l / \mathrm{g}\end{array}$ \\
\hline VIII & 293 & 2.21 & 67.0 & $5.45(5.89)^{2}$ \\
II & 157 & 2.33 & 30.7 & 3.71 \\
VII & 76.5 & 3.06 & 14.1 & 2.16 \\
V & 34.1 & 3.79 & 5.42 & 1.18 \\
III & 25.2 & 4.10 & 3.81 & 0.952
\end{tabular}

a Extrapolated to zero rate of shear (see the text). the data of Marzolph and Schulz (at $25^{\circ} \mathrm{C}$ ), ${ }^{15}$ and has pointed out that the plot has a weak concave curvature upward. In our case, however, the corresponding plots may be regarded as linear over the range studied, as seen from Figure 2. Deviations of the highest points from linearity in the figure may be regarded as arising from the fact that no shear-rate corrections were made. Therefore, we may estimate inversely zero-shear values of $[\eta]$ for sample VIII in good solvents from the lines in Figure 2 or eq 4. The numbers in parentheses in Tables I to III represent the values of $[\eta]$ thus obtained. For later analysis, we shall adopt these values.

Table IV summarizes light-scattering and intrinsic-viscosity data for sample VII in cyclohexane at various temperatures $t\left({ }^{\circ} \mathrm{C}\right)$ ranging from 32.2 to $60.1^{\circ} \mathrm{C}$. With these data, we plotted

Table III. Light-scattering and intrinsic-viscosity data for polystyrene in dichloroethane at $30^{\circ} \mathrm{C}$

\begin{tabular}{rcccl}
\hline Samples & $M_{w} \times 10^{-4}$ & $\begin{array}{c}A_{2} \times 10^{4}, \\
\mathrm{~m} l \mathrm{~mol} / \mathrm{g}^{2}\end{array}$ & $\begin{array}{c}\left\langle S^{2}\right\rangle \times 10^{12}, \\
\mathrm{~cm}^{2}\end{array}$ & $\begin{array}{c}{[\eta],} \\
\mathrm{d} l / \mathrm{g}\end{array}$ \\
\hline VIII & 284 & 1.86 & 59.8 & $4.69(4.97)^{\mathrm{a}}$ \\
II & 157 & 2.07 & 30.0 & 3.29 \\
VII & 77.4 & 2.65 & 12.5 & 1.91 \\
V & 34.1 & 3.18 & 4.96 & 1.04 \\
III & 24.8 & 3.41 & 3.41 & 0.824 \\
\hline
\end{tabular}

${ }^{a}$ Extrapolated to zero rate of shear (see the text).

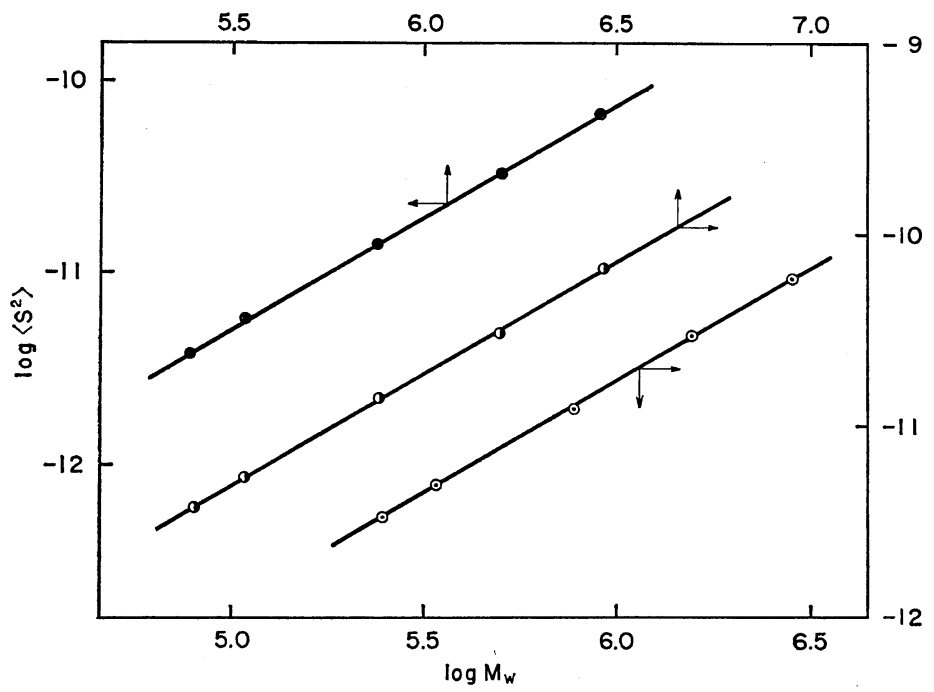

Figure 1. Double logarithmic plots of $\left\langle S^{2}\right\rangle$ against $M_{w}$ for polystyrene: $\mathbf{0}$, in benzene at $30^{\circ} \mathrm{C} ;$, in toluene at $30^{\circ} \mathrm{C}$; $\odot$, in dichloroethane at $30^{\circ} \mathrm{C}$. 


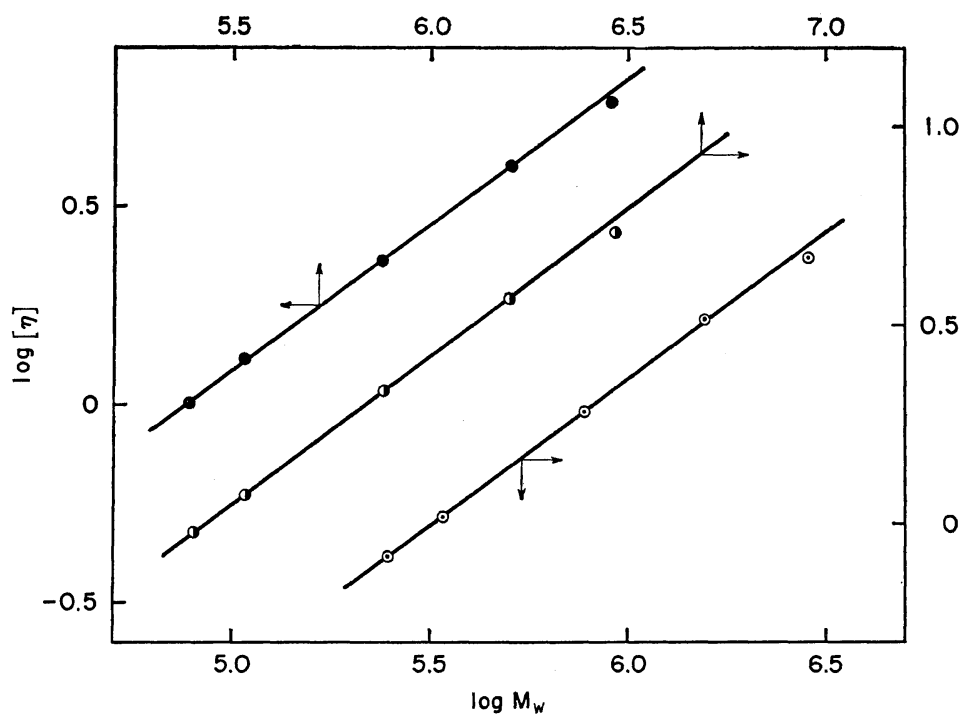

Figure 2. Double logarithmic plots of $[\eta]$ against $M_{w}$ for polystyrene. The symbols have the same significance as those in Figure 1.

Table. IV. Light-scattering and intrinsic-viscosity data for polystyrene sample VII in cyclohexane

\begin{tabular}{cccc}
\hline $\begin{array}{c}t, \\
{ }^{t} \mathrm{C}\end{array}$ & $\begin{array}{c}A_{2} \times 10^{4}, \\
\mathrm{~m} l \mathrm{~mol} / \mathrm{g}^{2}\end{array}$ & $\begin{array}{c}\left\langle S^{2}\right\rangle \times 10^{12} \\
\mathrm{~cm}^{2}\end{array}$ & $\begin{array}{c}{[\eta],} \\
\mathrm{d} l / \mathrm{g}\end{array}$ \\
\hline 60.1 & $0.93_{3}$ & 8.96 & 1.15 \\
50.1 & $0.67_{7}$ & 8.39 & 1.04 \\
45.4 & $0.55_{4}$ & 7.98 & 0.985 \\
40.2 & $0.31_{5}$ & 7.56 & 0.916 \\
37.3 & $0.18_{7}$ & 7.25 & 0.858 \\
35.2 & $0.08_{2}$ & 6.77 & 0.817 \\
34.2 & $-0.02_{5}$ & 6.51 & 0.793 \\
32.2 & $-0.18_{5}$ & 5.85 & 0.749
\end{tabular}

Table V. Mean-square radii and intrinsic viscosities in cyclohexane at the theta temperature $\left(34.6^{\circ} \mathrm{C}\right)$

\begin{tabular}{rlclc}
\hline Samples & $\begin{array}{c}\left\langle S^{2}\right\rangle_{0} \times 10^{12}, \\
\mathrm{~cm}^{2}\end{array}$ & $\begin{array}{c}\left\langle S^{2}\right\rangle_{0} / M_{w} \\
\times 10^{18}\end{array}$ & $\begin{array}{c}{[\eta]_{0}} \\
\mathrm{~d} l / g\end{array}$ & $\begin{array}{c}{[\eta]_{0} / M_{w^{1 / 2}} \times 10^{4}} \\
\times 1 / 2\end{array}$ \\
\hline VIII & 23.9 & 8.33 & 1.53 & 9.03 \\
II & - & - & 1.16 & 9.20 \\
VII & $6.61^{\text {a }}$ & 8.74 & $0.800^{\mathrm{a}}$ & 9.20 \\
V & 2.81 & 8.22 & 0.531 & 9.08 \\
III & - & - & 0.440 & 8.87 \\
\hline
\end{tabular}

a Interpolated values.

the product $A_{2} M_{w}$ against $t$, and found $\Theta=$ $34.6 \pm 0.2^{\circ} \mathrm{C}$ as the theta temperature. This value of $\Theta$ agrees, within experimental error, with the value of $34.8\left({ }^{\circ} \mathrm{C}\right)$ found by Orofino and Mickey ${ }^{16}$ for cyclohexane solutions of polystyrene prepared anionically in benzene.

Values of $\left\langle S^{2}\right\rangle_{0}$ and $[\eta]_{\theta}$ obtained in cyclohexane at $34.6{ }^{\circ} \mathrm{C}$ are given in Table $\mathrm{V}$, where the values for sample VII were obtained by interpolation, and the values of $M_{w}$ obtained in benzene were used. The values in the third and fifth columns allow us to assign the following values to the ratios $\left\langle S^{2}\right\rangle_{0} / M_{w}$ and $[\eta]_{\theta} / M_{w}{ }^{1 / 2}$

$$
\begin{aligned}
& \left\langle S^{2}\right\rangle_{0} / M_{w}=8.4 \times 10^{-18} \\
& {[\eta]_{\theta} / M_{w}{ }^{1 / 2}=9.1 \times 10^{-4}}
\end{aligned}
$$

For cyclohexane solutions of polystyrene prepared anionically in benzene ${ }^{8}$ Berry ${ }^{2}$ has assigned the values of $7.6 \times 10^{-18}$ and $8.4 \times 10^{-4}$ to these ratios, respectively, and Orofino and Mickey ${ }^{16}$ have obtained the value of $8.66 \times 10^{-4}$ for the latter ratio, though only for one sample. Although our value of $\left\langle S^{2}\right\rangle_{0} / M_{w}$ given by eq 5 is somewhat greater than the corresponding value assigned by Berry, the difference is not definitely conclusive, considering the scatter of observed values of this ratio about the assigned values in both cases. On the other hand, the ratio $[\eta]_{\theta} / M_{w}{ }^{1 / 2}$ can be estimated more accurately, and we may therefore corclude that our value given by eq 6 is definitely greater than the corresponding 
values assigned by Berry and also by Orofino and Mickey. To ascertain this conclusion, we carried out light-scattering and viscosity measurements on a fractionated polystyrene sample prepared by thermal polymerization, and obtained $[\eta]_{\theta} / M_{w}{ }^{1 / 2}=8.4 \times 10^{-4}$ with $M_{w}=126 \times 10^{4}$. Note that according to the earlier data obtained by Krigbaum and Flory $^{17}$ for polystyrene prepared by bulk polymerization with an initiator, $[\eta]_{\theta} / M_{v}^{1 / 2}=8.2 \times 10^{-4}$, where $M_{v}$ is the viscosityaverage molecular weight.

If we use the values given by eq 5 and 6 , we obtain the value of $2.5 \times 10^{21}$ for the FloryFox viscosity constant $\Phi_{0}$ at the theta temperature. On the other hand, Berry's assigned values give $\Phi_{0}=2.7 \times 10^{21}$, which may be regarded as close to our value. We calculated values of $\alpha_{S}$ according to the same procedure as before, ${ }^{1,5}$ i.e., on the basis of eq 5 . All values of $\alpha_{\eta}$ were calculated from the equation $\alpha_{\eta}{ }^{3}=[\eta] /[\eta]_{\theta}$ with observed values of $[\eta]_{\theta}$ without assuming eq 6.

\section{DISCUSSION}

\section{Samples}

The results obtained in the last section suggest that our polystyrenes prepared anionically in tetrahydrofuran may possibly differ from those prepared in benzene, in the local microstructure of the chain. However, the values of $\Theta$ found for the two series of samples coincide within experimental error. This means that there is no remarkable difference in the excludedvolume interaction between these samples, since at the $\Theta$ temperature the binarycluster integral $\beta$ vanishes within the framework of the twoparameter theoretical description. Furthermore, the obtained constancies of the ratios $[\eta]_{\theta} / M_{w}{ }^{1 / 2}$ and $\left\langle S^{2}\right\rangle_{0} / M_{w}$ are within experimental uncertainty. Therefore, our samples uesd may be regarded as homologous, and the obtained data can be employed to test the theory of the excluded-volume effect in dilute polymer solutions.

\section{Behavior of the Scattering Function}

It has recently been pointed out by many investigators $^{18,19}$ that Debye's scattering function $\boldsymbol{P}(x)$ is valid over a wide range for monodisperse chain polymers with or without excluded volume. $P(x)$ is given by ${ }^{20}$

$$
P(x)=\left(2 / x^{2}\right)\left(e^{-x}-1+x\right)
$$

where

$$
x=\left(16 \pi^{2} / \lambda^{2}\right)\left\langle S^{2}\right\rangle \sin ^{2}(\theta / 2)
$$

with $\lambda$ the wavelength of light in the solution. On the basis of this fact, Kato, et al., ${ }^{6}$ have determined $\left\langle S^{2}\right\rangle$ in good solvents to force agreement between observed and theoretical values of $P(x)$. Thus, for the present purpose, it is necessary to examine whether our values of $\left\langle S^{2}\right\rangle$ obtained by the method of square-root plots do or do not satisfy Debye's function.

In Figure 3 are plotted observed values of $P^{-1}(x)$ against $x$ with the values of $\left\langle S^{2}\right\rangle$ given in the tables for samples VIII, II, and VII in good solvents at $30^{\circ} \mathrm{C}$ and for sample VII in cyclohexane at $35.2^{\circ} \mathrm{C}$. The full curve represents the theoretical values. It is seen that the data points fit the theoretical curve very well over a wide range of $x$ but deviate downward from the curve for large $x$. This result has been observed by many investigators. ${ }^{6,18,19}$ In particular, the deviation is seen to occur for $x>c a .9$, and this is in agreement with the conclusion reached by Utiyama, et al.,$^{19}$ in much more precise measurements. The result displayed in Figure 3 indicates that there is no essential difference between our and Kato's procedures of determining $\left\langle S^{2}\right\rangle$; application of the latter procedure would give almost the same values of $\left\langle S^{2}\right\rangle$ as those given in the tables. Furthermore, the fit of our data to Debye's function suggests that our samples are fairly monodisperse.

\section{Behavior of $\Psi$}

We are now in a position to proceed to test the two-parameter theory with the present data. We first consider the behavior of the function $\Psi$. Its values calculated from eq 1 with observed values of $M_{w}$ (for $M$ ), $A_{2}$ and $\left\langle S^{2}\right\rangle$ given in the tables are plotted against the corresponding values of $\alpha_{S}{ }^{3}$ in Figure 4. The three curves represent the values predicted by the indicated theories, the attached symbols having the same meaning as before. ${ }^{1,5}$ That is, the symbol F, o means the original Flory-Krigbaum-Orofino theory ${ }^{21,22}$ of $\Psi$ combined with the original Flory theory ${ }^{23}$ of $\alpha_{S}, F, m$ the modified Flory- 
Krigbaum-Orofino theory ${ }^{21,22,24}$ of $\Psi$ combined with the modified Flory theory ${ }^{23,24}$ of $\alpha_{\mathrm{S}}$, and $Y$ the Kurata-Yamakawa theory ${ }^{25,26}$ of $\Psi$ combined with the Yamakawa-Tanaka theory ${ }^{27}$ of
$\alpha_{\mathrm{S}}$. The present data are seen to be consistent with those previously obtained for $\operatorname{poly}(p$ methylstyrene $)^{5}$ and $\operatorname{poly}(p$-bromostyrene $),{ }^{1}$ belonging to the first group stated in the Introduc-

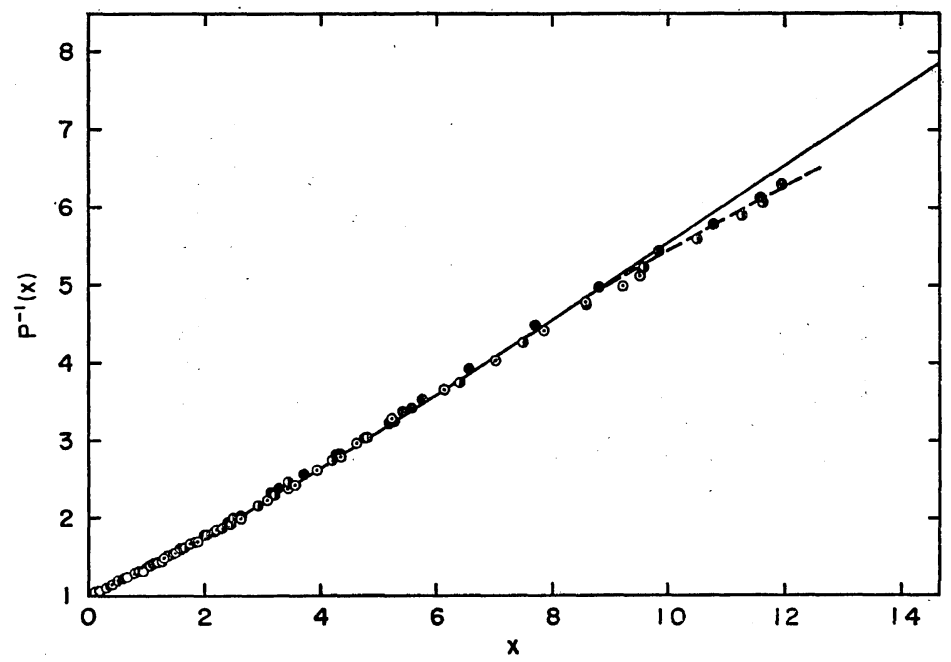

Figure 3. The reciprocal scattering function plotted against the reduced variable $x$ defined by eq 8 for samples VIII, II, and VII: 0 , in benzene at $30^{\circ} \mathrm{C}$; , in toluene at $30^{\circ} \mathrm{C} ; \odot$, in dichloroethane at $30^{\circ} \mathrm{C}$; and for sample VII: $\bigcirc$, cyclohexane at $35.2^{\circ} \mathrm{C}$. The full curve represents the values predicted by Debye's function.

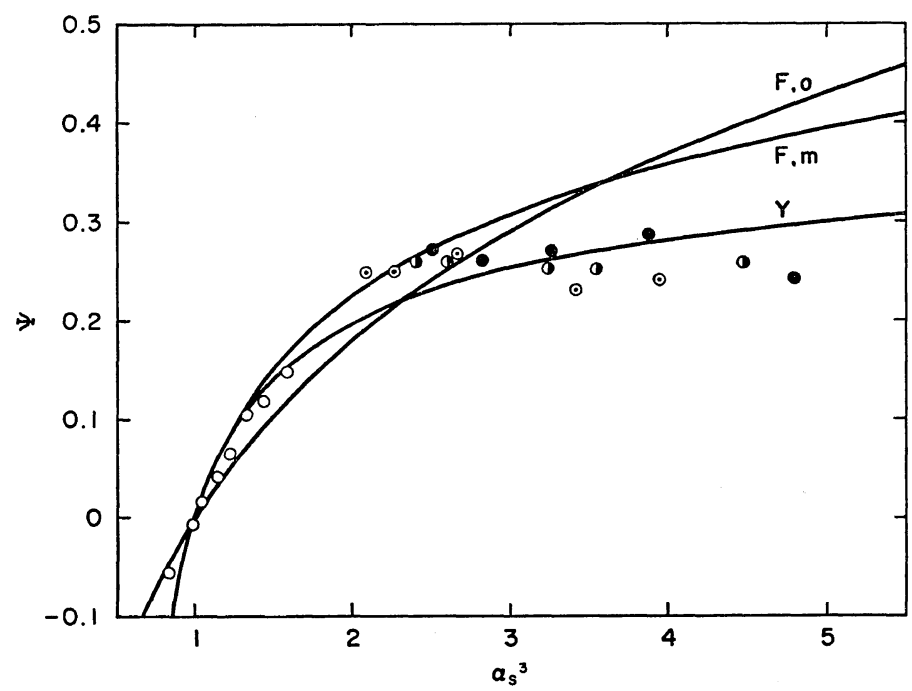

Figure 4. Plots of $\Psi$ against $\alpha \mathrm{S}^{3}$ for polystyrene: in benzene at $30^{\circ} \mathrm{C}$; in toluene at $30^{\circ} \mathrm{C} ; \odot$, in dichloroethane at $30^{\circ} \mathrm{C} ; \bigcirc$, sample VII in cyclohexane at various temperatures. The curves represent the theoretical values: curve $F$, $o$, the original Flory-Krigbaum-Orofino theory of $\Psi$ with the original Flory theory of $\alpha_{\mathrm{S}}$; curve $\mathrm{F}, \mathrm{m}$, the modified Flory-Krigbaum-Orofino theory of $\Psi$ with the modified Flory theory of $\alpha_{\mathrm{S}}$; curve Y, the Kurata-Yamakawa theory of $\Psi$ with the Yamakawa-Tanaka theory of $\alpha_{S}$. 


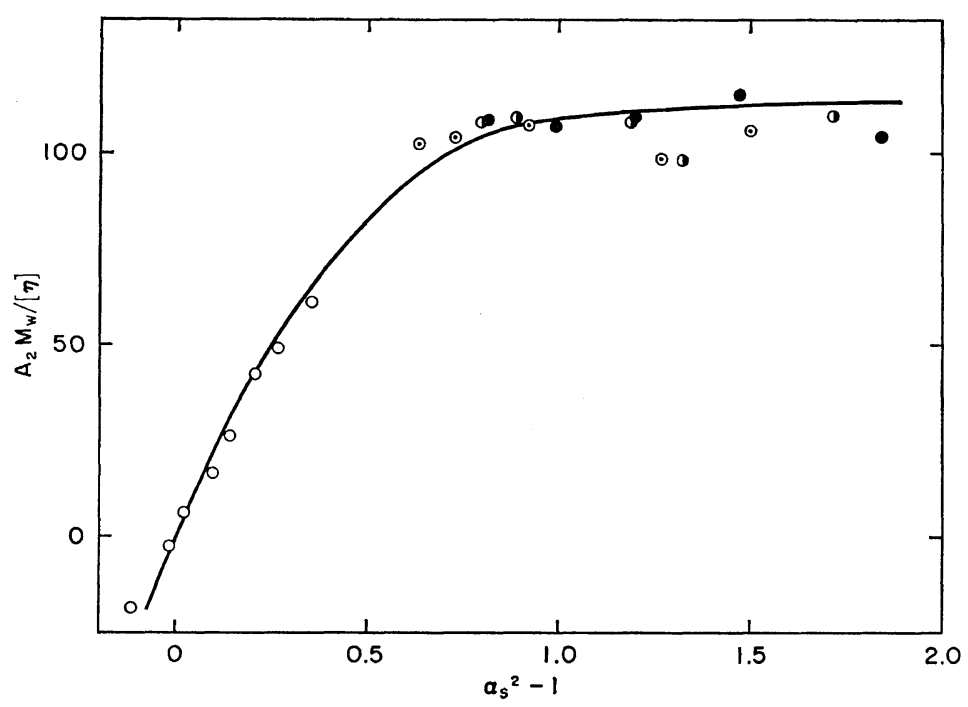

Figure 5. Plots of $A_{2} M_{w} /[\eta]$ against $\alpha_{\mathrm{s}}{ }^{2}-1$ for polystyrene. The symbols have the same significance as those in Figure 4. The full curve represents the best fit to the data for polychloroprene ${ }^{4}$ and $\operatorname{poly}\left(p\right.$-methylstyrene).$^{5}$

tion. It is important to observe that that the values of $\Psi$ reach $0.25-0.30$ in good solvents.

The corresponding values of $\Psi$ obtained by Kato, et al., ${ }^{6}$ for poly( $\alpha$-methylstyrene) in toluene are only about 0.2 . This difference may be considered to arise mainly from the fact that their values of $\left\langle S^{2}\right\rangle^{3 / 2}$ are about $2.5 / 2.0$ times as large as our corresponding values, since the ratio of their value of $\Phi_{0}$ to ours is about 2.0/2.5. Another reason for the difference is that Kato's values of $A_{2}$ may probably be a little smaller than our corresponding values. However, it must be recalled that there is no essential difference between our and their methods of determining $\left\langle S^{2}\right\rangle$, as already discussed. Further, Kato, et $a l$., also applied that method of squareroot plots to determine $A_{2}$, as was done by us.

In this connection, values of the dimensionless ratio $A_{2} M_{w} /[\eta]$, which behaves like $\Psi$, are plotted against the corresponding values of $\alpha_{\mathrm{S}}{ }^{2}-1$ in Figure 5. The curve represents the best fit to the data for polychloroprene $e^{3,4}$ and $\operatorname{poly}(p$ methylstyrene). ${ }^{5} \quad$ The relationship determined previously is seen to be well reproduced.

\section{Behavior of $\alpha_{\eta}$}

Figure 6 shows plots of $\log \alpha_{\eta}{ }^{3}$ against $\log \alpha_{\mathrm{s}}{ }^{3}$. The curve represents the best fit to the data for polychloroprene ${ }^{4}$ and poly $(p$-bromostyrene $) .{ }^{1}$ The present data are seen to be consistent with the previous results. However, it must be noted that the values of $\log \alpha_{\eta}{ }^{3}$ for poly ( $p$-methylstyrene) in toluene $\mathrm{e}^{5}$ are a little too large for $\log \alpha_{\mathrm{S}}{ }^{3}=$ 0.4-0.6 compared to the results displayed in the figure. To show the situation more clearly, values of the ratio of the viscosity constant to its unperturbed value calculated from the equation, $\Phi / \Phi_{0}=\alpha_{\eta}{ }^{3} / \alpha_{\mathrm{S}}{ }^{3}$, are plotted against the corresponding values of $\alpha_{\mathrm{S}}{ }^{3}$ in Figure 7 . Curve 1 represents the best fit to the data for polychloroprene $^{4}$ and poly( $p$-methylstyrene), ${ }^{5}$ and curve 2 the best fit to the data for poly $(p$ bromostyrene $)^{1}$ and the present data. All the results obtained previously and in the present investigation are seen to be consistent with one another for $\alpha_{\mathrm{S}}{ }^{3}<2.5$, while the values of $\Phi / \Phi_{0}$ for poly ( $p$-methylstyrene) in toluene, ${ }^{5}$ for which $\alpha_{\mathrm{S}}{ }^{3}$ lies between 2.5 and 3.7 , are a little too large. This may be regarded as arising from experimental uncertainty.

Figure 8 shows plots of $\alpha_{\eta}{ }^{3}$ against $z$, where the values of $z$ for the data points were determined from the values of $\alpha_{\mathrm{S}}$ using the Yamakawa-Tanaka equation, ${ }^{27}$

$$
\alpha_{\mathrm{S}}{ }^{2}=0.541+0.459(1+6.04 z)^{0.46}
$$

The curve represents the best fit to the data for 


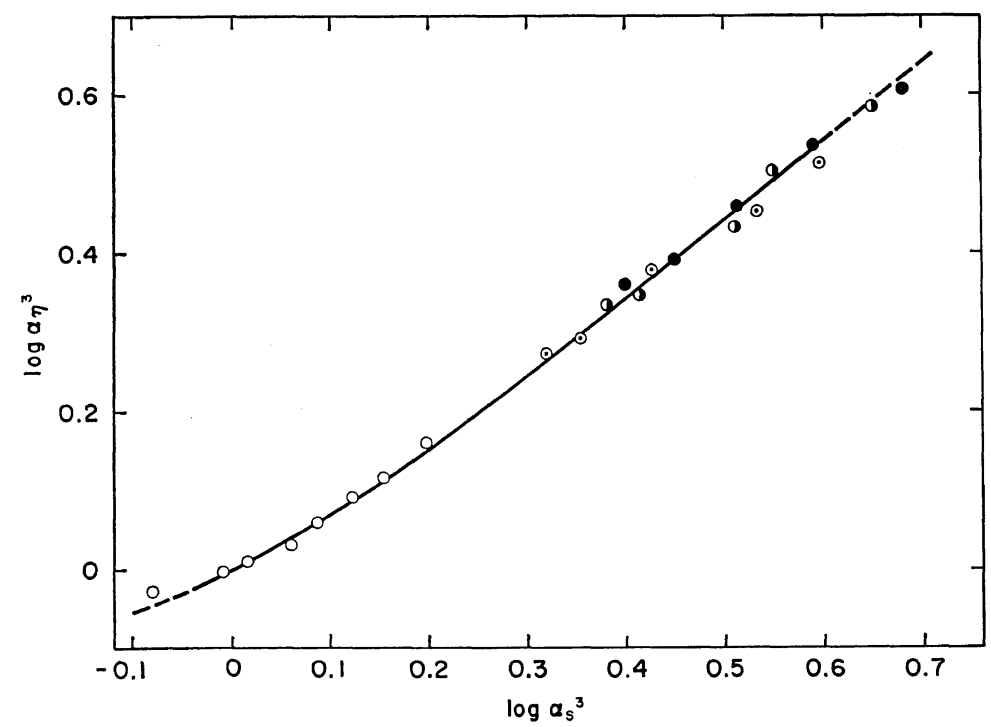

Figure 6. Double logarithmic plots of $\alpha_{\eta}{ }^{3}$ against $\alpha \mathrm{s}^{3}$ for polystyrene. The symbols have the same significance as those in Figure 4 . The curve represents the best fit to the data for polychloroprene ${ }^{4}$ and poly( $p$-bromostyrene $){ }^{1}$

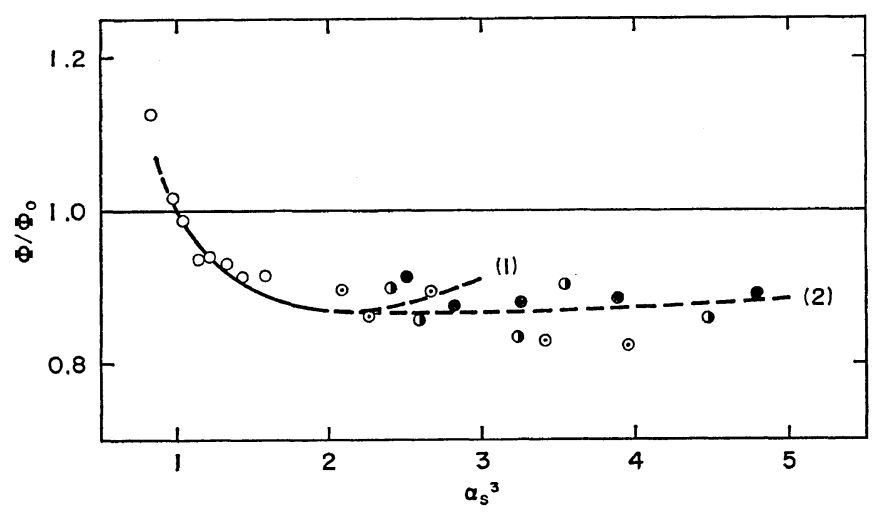

Figure 7. Plots of $\Phi / \Phi_{0}$ against $\alpha_{\mathrm{S}}{ }^{3}$ for polystyrene. The symbols have the same significance as those in Figure 6: curve 1, the best fit to the data for polychloroprene $e^{4}$ and $\operatorname{poly}(p$-methylstyrene $) ;{ }^{5}$ curve 2 , the best fit to the date for poly $(p \text {-bromostyrene })^{1}$ and the present data.

polychloroprene ${ }^{4}$ and poly $\left(p\right.$-bromostyrene). ${ }^{1}$ The relationship is well reproduced, indicating that $\alpha_{\eta}{ }^{3}$ is a function of only $z$. The insert of Figure 8 is an enlargement of the region of small $z$. Straight line 1 represents the first-order perturbation theory prediction recently obtained by Yamakawa and Tanaka ${ }^{28}$

$$
\alpha_{\eta}{ }^{3}=1+C_{1} z-\cdots
$$

with $C_{1}=1.06$, and straight line 2 the earlier prediction with $C_{1}=1.55$ obtained by Kurata and Yamakawa. ${ }^{29}$ The new theory is seen to be in close agreement with experiment. A detailed discussion of the range of validity of the first-order perturbation theory of $\alpha_{\eta}{ }^{3}$ has already been given in the previous paper, ${ }^{1}$ and we do not repeat it here.

As shown previously, ${ }^{1}$ the data of Kato, et al. ${ }^{6}$ for $\operatorname{poly}(\alpha$-methylstyrene $)$ in toluene satisfy the Yamakawa-Tanaka equation 9 for $\alpha_{\mathrm{S}}$ rather 
A. Yamamoto, M. Fujil, G. Tanaka, and H. Yamakawa

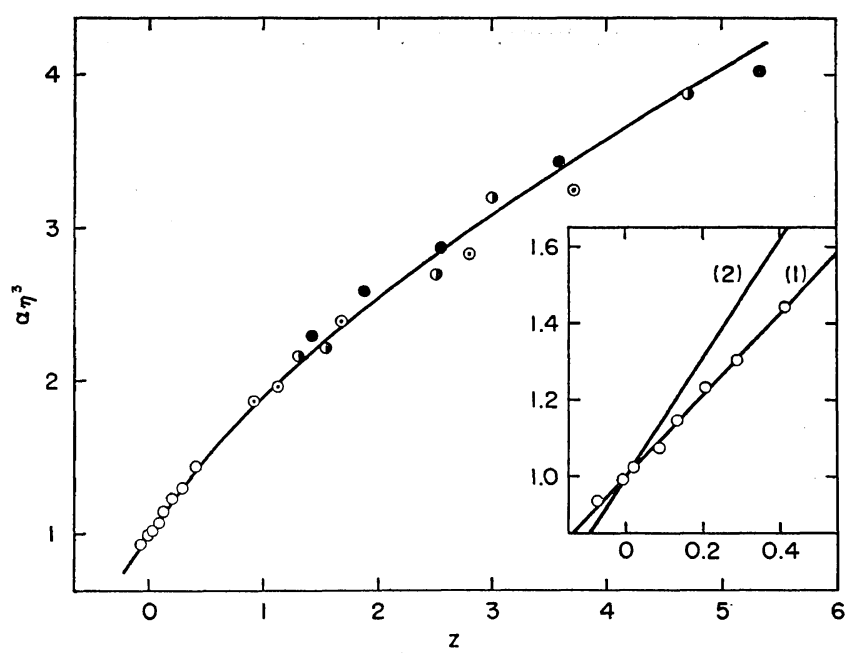

Figure 8. Plots of $\alpha_{\eta}{ }^{3}$ against $z$ for polystyrene, where the values of $z$ [were calculated from the values of $\alpha_{\mathrm{S}}$ using eq 9. The symbols have the same significance as those in Figure 6 . The curve represents the best fit to the data for polychloroprene ${ }^{4}$ and poly ( $p$-bromostyrene). ${ }^{1}$ The insert is an enlargement of the region of small $z$ : line 1, the first-order perturbation theory of Yamakawa and Tanaka (eq 10) with $C_{1}=1.06$; line 2 , the Kurata-Yamakawa theory with $C_{1}=1.55$.

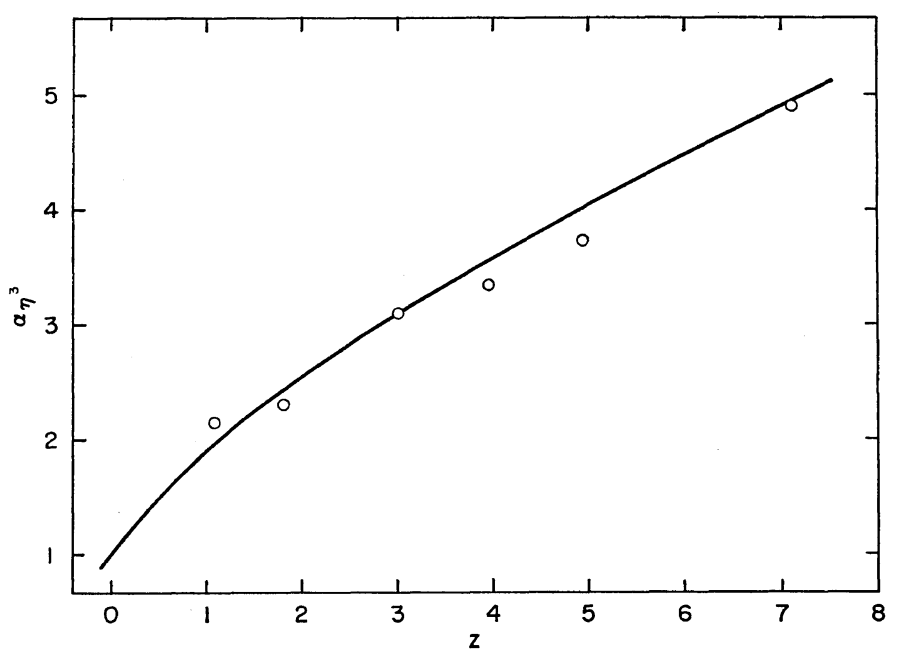

Figure 9. Plots of $\alpha_{\eta}^{3}$ against $z$ for Kato's poly( $\alpha$-methylstyrene) in toluene, 6,7 where the values of $z$ were calculated from the values of $\alpha_{\mathrm{S}}$ using eq 9. The curve represents the best fit to the data, as in Figure 8.

than the modified Flory equation. Therefore, we re-estimated values of $z$ for this case by the use of eq 9 as in our analysis, and their values of $\alpha_{\eta}{ }^{3}$ are plotted against the values of $z$ thus determined in Figure 9. The curve represents the same as that in Figure 8. It is seen that their values of $\alpha_{\eta}{ }^{3}$ in toluene satisfy within experimental error the relationship between $\alpha_{\eta}{ }^{3}$ and $z$ established by us. Further, we note that the data of Kato, et al., for theta-solvent systems fall on and near the curve in Figure 9. Thus we may conclude that according to our procedure 


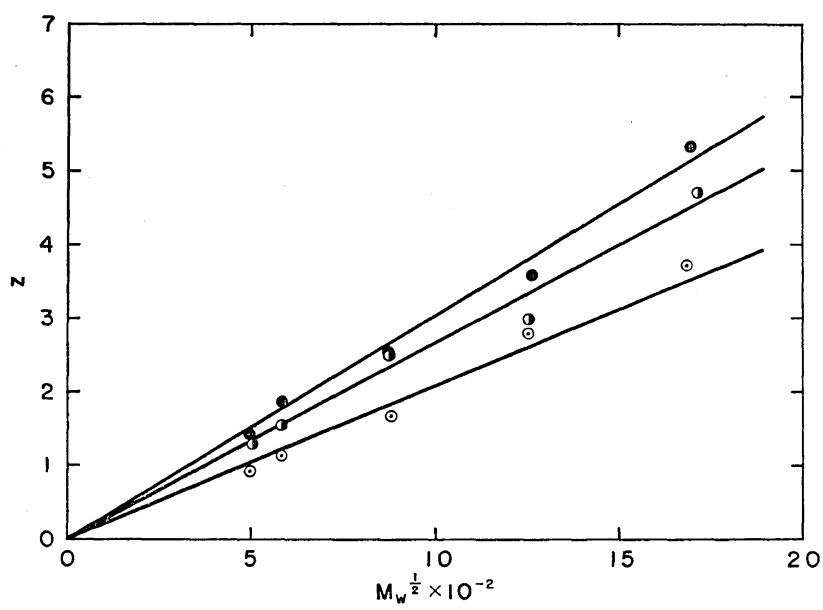

Figure 10. Plots of $z$ against $M_{w}^{1 / 2}$ for polystyrene: in benzene at $30^{\circ} \mathrm{C}$; in toluene at $30^{\circ} \mathrm{C} ; \odot$, in dichloroethane at $30^{\circ} \mathrm{C}$. The values of $z$ were calculated from the values of $\alpha_{\mathrm{S}}$ using eq 9.

of analyzing data, their data also form a single-composite curve of $\alpha_{\eta}{ }^{3}$ against $z$ which is consistent with ours.

\section{Binary-cluster Integral}

We have used the dependence of $\Psi$ on $\alpha_{\mathrm{s}}{ }^{3}$ as one criterion to test the two-parameter theory in the previous and present studies. Another criterion is the proportionality of $z$ to $M^{1 / 2}$. Values of $z$ in good solvents determined from the values of $\alpha_{\mathrm{S}}$ using eq 9 are plotted against $M_{w}{ }^{1 / 2}$ in Figure 10. The data points fall near the straight lines passing through the origin, as expected.

Now, from the slopes of these straight lines, we can estimate $\beta$ in good solvents, or more strictly the interaction parameter $B$ defined by

$$
B=(n / M)^{2} \beta
$$

since eq 2 may be rewritten in the form

$$
z / M^{1 / 2}=\left(4 \pi\left\langle S^{2}\right\rangle_{0} / M\right)^{-3 / 2} B
$$

The determination of $\beta$ depends on the choice of $n$, which is, to some extent, arbitrary within the framework of the two-parameter theory. However, if we take a monomeric unit as a segment, for convenience, then we obtain the values of $\beta$ given in the third column of Table VI, where the value of $\left\langle S^{2}\right\rangle_{0} / M$ given by eq 5 was adopted. In the same column are also given the values of $\beta$ in cyclohexane at various
Table VI. Values of $\beta$ per monomeric unit for polystyrene in various solvent

\begin{tabular}{lccc}
\hline \multirow{2}{*}{ Solvent } & Temperature, & \multicolumn{2}{c}{$\beta \times 10^{24}\left(\mathrm{~cm}^{2}\right)$} \\
\cline { 2 - 4 } & & From $\alpha_{\mathrm{S}}$ & From $[\eta]$ \\
\hline Benzene & 30 & 35.6 & 34.2 \\
Toluene & 30 & 31.2 & 29.4 \\
Dichlorcethane & 30 & 24.4 & 22.7 \\
Cyclohexane & 60.1 & 5.89 & 6.06 \\
& 50.1 & 4.10 & 4.18 \\
& 45.4 & 2.93 & 3.19 \\
& 40.2 & 1.88 & 2.01 \\
& 37.3 & 1.23 & 1.02 \\
& 35.2 & 0.28 & 0.32 \\
& 34.2 & -0.14 & $-0.08_{6}$ \\
& 32.2 & -1.08 & -0.83 \\
\hline
\end{tabular}

temperatures obtained in a similar manner from the values of $\alpha_{\mathrm{S}}$ for sample VII.

Values of $\beta$ can also be determined from intrinsic viscosities, though in a crude fashion, as discussed previously. ${ }^{5}$ The method is based upon the modified Stockmayer-Fixman equations, ${ }^{5,30}$

$[\eta] / M^{1 / 2}=K+0.346 \Phi_{0} B M^{1 / 2}$ for $0<\alpha_{\eta}^{3}<1.6$

$[\eta] / M^{1 / 2}=1.05 K+0.287 \Phi_{0} B M^{1 / 2}$ for $0<\alpha_{\eta}^{3}<2.5$

with $K=[\eta]_{\theta} / M^{1 / 2}$. We note that eq 13 is 


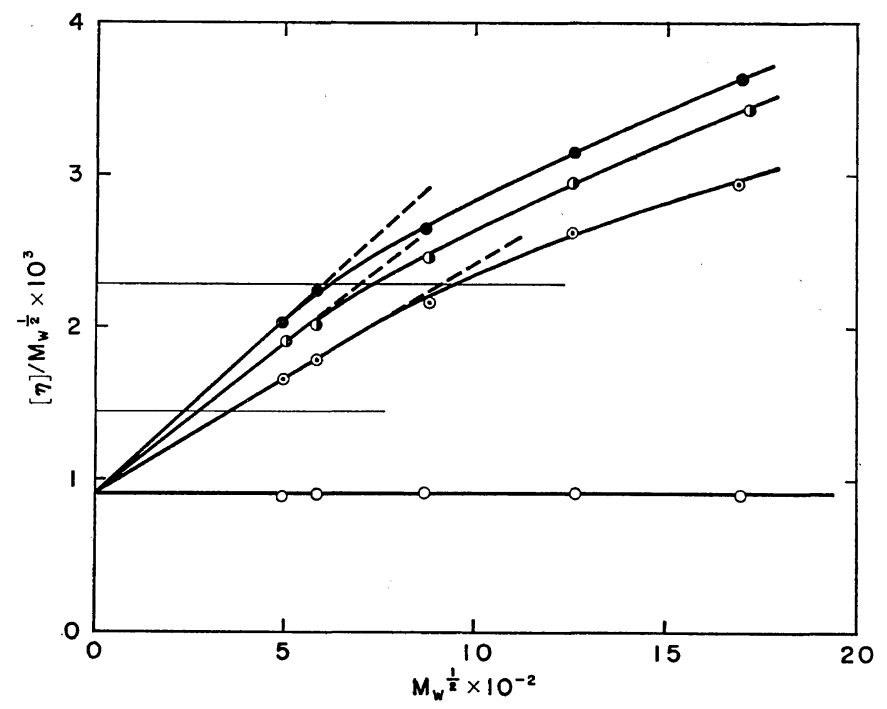

Figure 11. Stockmayer-Fixman plots for polystyrene: $\bullet$, in benzene at $30^{\circ} \mathrm{C}$; (), in toluene at $30^{\circ} \mathrm{C}$; $\odot$, in dichloroethane at $30^{\circ} \mathrm{C} ; \bigcirc$, in cyclohexane at $34.6^{\circ} \mathrm{C}$ $(\Theta)$. The broken lines are the initial tangents. The two thin horizontal lines indicate the upper bounds given in eq 13 and 14.

formally equivalent to eq 10 with $C_{1}=1.05$, instead of $C_{1}=1.06$, and therefore that it does not exactly correspond to the first-order perturbation theory of $\alpha_{\eta}^{3}$ but is only semiempirical. According to eq 13 and 14 , values of $[\eta] / M_{w}{ }^{1 / 2}$ in good solvents and in cyclohexane at the $\Theta$ temperature are plotted against $M_{w}^{1 / 2}$ in Figure 11. The two thin horizontal lines in the figure indicate the upper bounds below which eq 13 and 14 are valid, corresponding to $[\eta] / K M_{w}{ }^{1 / 2}=$ 1.6 and 2.5, respectively. For good-solvent systems extrapolations to $M_{w}=0$ were carried out to give the same intercept as that in cyclohexane, ignoring the minor difference between $K$ and $1.05 K$. The broken lines in the figure indicate the tangents to those parts of the curves which may be regarded as linear. Values of $B$ and therefore $\beta$, were determined from the slopes of these broken lines using eq 14 . The results are given in the fourth column of Table VI. In the same column are also given the values of $\beta$ in cyclohexane at various temperatures obtained from the values of $[\eta]$ for sample VII using eq 13. The values of $\beta$ obtained by the two methods are seen to agree with each other within experimental uncertainty.

A simple analysis of the excess binary-cluster integral for polar polymers has been given in the previous paper. ${ }^{31}$ However, a theoretical treatment of the whole $\beta$, as obtained in the present study for nonpolar polymers, involves many problems to be considered. Some preliminary results will be reported in another paper. ${ }^{32}$

\section{CONCLUSION}

Experimental tests of the theory of the excluded-volume effect in dilute polymer solutions were made using light-scattering and viscosity data obtained for monodisperse polystyrenes prepared anionically in tetrahydrofuran. Our samples yielded a value of $K$ somewhat greater than the corresponding value for Berry's samples prepared anionically in benzene. This suggests that the two types of samples differ in microstructure, though the precise difference is unknown. However, it has been shown that the relationships or two-parameter description established experimentally in previous papers are well reproduced in the present data, indicating that these also belong to the first group according to the classification stated in the Introduction.

Although the data of Kato, et al., belong to the second group, it has been shown that their 
values of $\alpha_{\mathbb{S}}$ and $\alpha_{\eta}$ are consistent with our corresponding values with respect to the $z$ dependences if reanalysis of the data is made according to our procedure. The only differences consist in the values of $\Phi_{0}$ and $\Psi$. If we consider the fact that there is no essential difference between our and their methods of determining $A_{2}$ and $\left\langle S^{2}\right\rangle$, it seems unlikely that the differences in $\Phi_{0}$ and $\Psi$ are related to measurements and analysis of the data. We tend to believe the difference is associated with and reflected in the samples prepared anionically by Kato, et al.

\section{REFERENCES}

1. K. Takashima, G. Tanaka, and H. Yamakawa, Polymer J., 2, 245 (1971).

2. G. C. Berry, J. Chem., Phys., 44, 4550 (1966); 46, 1338 (1967).

3. T. Norisuye, K. Kawahara, A. Teramoto, and H. Fujita, ibid., 49, 4330 (1968).

4. K. Kawahara, T. Norisuye, and H. Fujita, ibid., 49, 4339 (1968).

5. G. Tanaka, S. Imai, and H. Yamakawa, ibid., 52, 2639 (1970).

6. T. Kato, K. Miyaso, I. Noda, T. Fujimoto, and M. Nagasawa, Macromolecules, 3, 777 (1970).

7. I. Noda, K. Mizutani, T. Kato, T. Fujimoto, and M. Nagasawa, ibid., 3, 787 (1970).

8. F. Wenger and S.-P. S. Yen, Makromol. Chem., 43, 1 (1961).

9. M. Morton, R. Milkovich, D. B. McIntyre, and L. J. Bradley, J. Polym. Sci., Part A, 1, 443 (1963).

10. M. Morton, A. A. Rembaum, and J. L. Hall, ibid., Part A, 1, 461 (1963).

11. "International Critical Tables," Vol. III, E. W. Washburn, Ed., McGraw-Hill Book Co., New
York, N.Y., 1928, pp 28 and 29.

12. C. I. Carr and B. H. Zimm, J. Chem. Phys., 18, 1616 (1950).

13. D. K. Carpenter and W. R. Krigbaum, ibid., 24, 1041 (1956).

14. H. Fujita, Polymer J., 1, 537 (1970).

15. H. Marzolph and G. V. Schulz, Makromol. Chem., 13, 120 (1954).

16. T. A. Orofino and J. W. Mickey, J. Chem. Phys., 38, 2512 (1963).

17. W. R. Krigbaum and P. J. Flory, J. Polym. Sci., 11, 37 (1953).

18. T. E. Smith and D. K. Carpenter, Macromolecules, 1, 204 (1968).

19. H. Utiyama, Y. Tsunashima, and M. Kurata, J. Chem. Phys., to be published. See also the papers cited therein.

20. P. Debye, J. Phys. Colloid Chem., 51, 18 (1947).

21. P. J. Flory and W. R. Krigbaum, J. Chem. Phys., 18, 1086 (1950).

22. T. A. Orofino and P. J. Flory, ibid., 26, 1067 (1957).

23. P. J. Flory, ibid., 17, 303 (1949).

24. W. H. Stockmayer, Makromol. Chem., 35, 54 (1960).

25. M. Kurata, M. Fukatsu, H. Sotobayashi, and H. Yamakawa, J. Chem. Phys., 41, 139 (1964).

26. H. Yamakawa, ibid., 48, 2103 (1968). See also Ref. 1.

27. H. Yamakawa and G. Tanaka, ibid., 47, 3991 (1967).

28. H. Yamakawa and G. Tanaka, ibid., in press.

29. M. Kurata and H. Yamakawa, ibid., 29, 311 (1958).

30. W. H. Stockmayer and M. Fixman, J. Polym. Sci., Part C, 1, 137 (1963).

31. Y. Noguchi, A. Aoki, G. Tanaka, and H. Yamakawa, J. Chem. Phys., 52, 2651 (1970).

32. H. Yamakawa and M. Fujii, ibid., in press. 